\title{
COMPORTAMIENTO DEL VIENTO LOCAL DIURNO EN LA CUENCA DEL RÍO LLOBREGAT
}

\author{
Jordi Comalrena de Sobregrau Bailina \\ Oficina Tècnica de Prevenció Municipal d'Incendis Forestals \\ Diputació de Barcelona
}

\section{RESUMEN}

El estudio de los datos meteorológicos de cinco estaciones representativas de la cuenca del río Llobregat (veranos de 2006, 2007 y 2009) para establecer patrones de comportamiento del viento local diurno que puedan ayudar a las tareas de prevención de incendios forestales ha revelado que la marinada es el elemento principal a tener en cuenta sólo en la zona costera y hasta la sierra Prelitoral. El patrón de la zona media y alta de la cuenca (interior) responde, en cambio, a la influencia del viento de valle y los vientos de ladera. El sistema de vientos locales de la cuenca del Llobregat supone un factor añadido de riesgo meteorológico de propagación de incendios forestales, dado que la velocidad más alta se detecta durante el período de mayor riesgo: a la temperatura elevada y a la baja humedad hay que sumar, por lo tanto, la velocidad del viento máxima diaria. Sin embargo, la previsibilidad del sistema, la velocidad media baja o moderada y la ausencia de cambios bruscos e imprevistos de velocidad y dirección descartan considerarlo un elemento de importante riesgo por sí mismo.

Palabras clave: vientos locales, Llobregat, prevención de incendios forestales, riesgo meteorológico, marinada, brisa marina.

\begin{abstract}
A study of meteorological data from five stations representative of the Llobregat River basin (summers of 2006, 2007 and 2009) was conducted in order to determine local daytime wind behavior patterns, an understanding of which may assist in forest fire prevention planning. Results reveal that the sea breeze is the
\end{abstract}


principal element to be considered when looking specifically at the area extending from the coastal zone to the pre-coastal range. However, the pattern in the middle and high zones of the basin (interior) is influenced by along-valley and downslope winds. The local wind patterns in the Llobregat basin constitute an additional meteorological risk factor related to the spread of forest fires. Maximum wind speeds are detected when other key risk factors like high temperature and low humidity are present, thus contributing to the total risk. However, the predictability of the system, low or moderate average wind speed and the absence of brusque and unexpected changes in wind velocity and direction, make the wind system an unsatisfactory risk factor in and of itself.

Key words: local winds, Llobregat, forest fire prevention, meteorological risk, sea breeze.

\section{INTRODUCCIÓN}

En este estudio se analizan los datos de cinco estaciones meteorológicas representativas de la cuenca del río Llobregat (Viladecans, El Pont de Vilomara, Berga, Òdena y Clariana de Cardener) para establecer patrones de comportamiento del viento local diurno que puedan ayudar a las tareas de prevención de incendios forestales.

El riesgo de propagación de un incendio se multiplica cuando determinadas situaciones sinópticas llegan acompañadas de viento. En Cataluña, las situaciones de sur, poniente y norte son las que se han relacionado con los grandes incendios forestales, y cada una de ellas presenta peculiaridades sinópticas que la distinguen de los días típicos de verano (Montserrat, 1998).

La época estival se caracteriza generalmente por situaciones de estabilidad y calma. La situación sinóptica más recurrente en verano en el Mediterráneo Occidental es el pantano barométrico, caracterizado por un gradiente de presión mínimo y muy poco movimiento en superficie, y que favorece el rol de los vientos de carácter local, básicamente los sistemas brisa marina - terral, viento de valle - montaña y los vientos de ladera anabáticos o ascendentes y catabáticos o descendentes.

La brisa marina es un viento típico del litoral, sobre todo durante la mitad cálida del año. Se produce cuando el aire que hay sobre la costa se calienta más rápidamente que el que hay sobre el mar, mediante la actividad solar. El aire sobre la costa se hace menos denso y tiende a elevarse, y para ocupar ese vacío se crea una corriente de aire desde el mar hacia la costa. De noche, el proceso se invierte y surge el terral, que sopla desde tierra adentro hacia el mar debido a un mayor enfriamiento del aire sobre la costa que del que hay sobre el mar. En el interior, se desarrolla un proceso parecido al del sistema 
marinada - terral cuando el sol calienta las laderas de los valles orientadas al sur y al este. El aire que hay sobre ésas también tiende a elevarse y se crean los vientos de ladera, que ascienden des del fondo de valle en dirección a las cumbres. Empiezan a soplar poco después de la salida del sol, y unas horas más tarde, bajo las mismas condiciones y cuando los rayos solares ya calientan el fondo de valle, aparece el viento de valle, que asciende por la cuenca, resiguiendo en sentido contrario el río. De noche, la inversión del proceso conlleva la aparición de los vientos de ladera descendientes y el viento de montaña, río abajo.

Los vientos locales influyen en el aumento del riesgo de propagación de un incendio; en especial los diurnos, mucho más intensos que los nocturnos (Prohom, 1998), de modo que los incendios topográficos (aquellos en los que el factor dominante es la dinámica local de vientos de convección) se propagan con una alta intensidad diurna y una baja intensidad nocturna (Castellnou et al., 2009), suponiendo un $21 \%$ de la superficie quemada en Cataluña en incendios con un patrón de propagación conocido (Castellnou et al., 2009). A pesar de eso, «las circulaciones de vientos locales han sido escasamente investigadas por el colectivo de geógrafos y físicos españoles dedicados al estudio del clima (Azorín Molina, 2004: 19-20)». Por esos dos motivos se justifica el análisis, básicamente de los diurnos, de cara a valorar si se dan patrones de comportamiento que puedan ayudar a las tareas de prevención.

\section{METODOLOGÍA}

\subsection{Zona de estudio}

La zona de estudio comprende la cuenca del río Llobregat (incluidos los afluentes Cardener y Anoia), interesante por ser la principal cuenca fluvial de la provincia de Barcelona y por incluir las dos comarcas más problemáticas a nivel de incendios forestales: Anoia y Bages. El uso de esta unidad geográfica se explica porque se cree que puede facilitar el análisis de los resultados, dado que la dirección y la velocidad del viento dependen mucho del relieve y la orografía locales.

Dentro de la cuenca hay veinte estaciones del Servei Meteorològic de Catalunya, y dos más que no pertenecen estrictamente a ella, aunque la distancia con la divisoria de aguas es mínima (Viladecans y Sant Llorenç Savall).

En un estudio preliminar, se analizaron los datos meteorológicos del verano de 2009 de todas las estaciones, a excepción de Gisclareny, directamente descartada por su altitud y su posición pirenaica y poco representativa. También se descartaron las estaciones de Artés y Vacarisses debido a la ausencia de registros 
de velocidad y dirección del viento. Además, se concluyó que no se podía definir un patrón habitual de comportamiento del viento local para las estaciones de Montmaneu (La Panadella), Monistrol de Montserrat, La Coma i la Pedra (Port del Comte) y Sant Llorenç Savall. Acto seguido, cuando se decidió continuar con el estudio alargando la serie temporal, se escogieron 5 estaciones de las 15 restantes, que fuesen representativas y con una serie temporal larga: Viladecans, la estación más próxima a la costa; El Pont de Vilomara, situada en el curso medio del río y representativa de la zona del Bages; Berga (Queralt), en el curso alto y en una zona elevada; Òdena, representante de la Anoia; y Clariana de Cardener, representativa de la zona del propio afluente.

Tabla 1. Localización y características de las cinco estaciones meteorológicas

\begin{tabular}{|c|c|c|c|c|c|c|c|}
\hline CÓDIGO & UTM_X & UTM_Y & MUNICIPIO & COMARCA & $\begin{array}{c}\text { ALTITUD } \\
(\mathbf{m})\end{array}$ & $\begin{array}{c}\text { DATOS } \\
(\mathbf{H} / \mathrm{S})\end{array}$ & $\begin{array}{c}\text { ALTURA } \\
\text { V }(\mathbf{m})\end{array}$ \\
\hline UG & 419600 & 4572540 & Viladecans & Baix Llobregat & 13 & $\mathrm{H}$ & 2 \\
\hline Rl & 406502 & 4614544 & $\begin{array}{c}\text { El Pont de } \\
\text { Vilomara i } \\
\text { Rocafort }\end{array}$ & Bages & 210 & $\mathrm{~S}$ & 10 \\
\hline WM & 403150 & 4662550 & $\begin{array}{c}\text { Berga (Santuari } \\
\text { de Queralt) }\end{array}$ & Berguedà & 1140 & $\mathrm{H}$ & 10 \\
\hline H1 & 387816 & 4604811 & Òdena & Anoia & 333 & $\mathrm{~S}$ & 10 \\
\hline CA & 382830 & 4645815 & $\begin{array}{c}\text { Clariana de } \\
\text { Cardener }\end{array}$ & Solsonès & 693 & $\mathrm{~S}$ & 10 \\
\hline
\end{tabular}

(Datos H: Horarios / S: Semihorarios. Altura V: altura de la medida de la velocidad del viento)

\subsection{Serie temporal}

Los datos tratados corresponden a los veranos (meses de junio, julio y agosto) de los años 2006, 2007 y 2009. Se decidió usar este periodo anual ya que, además de corresponder oficialmente al verano meteorológico, es la época con más incendios forestales y superficie quemada. En cuanto a la longitud de la serie, se estima que el uso de los datos de tres veranos es suficientemente representativo y tiene valor climatológico suficiente para poder establecer los patrones de comportamiento del viento local en las cinco estaciones estudiadas. Por último, se descartó analizar los datos del verano de 2008 a favor de 2006 debido a que el primero fue un verano atípico e inestable, con una superficie forestal quemada irrisoria en la provincia de Barcelona. 
Figura 1. Mapa de la cuenca del río Llobregat y localización de las cinco estaciones meteorológicas.

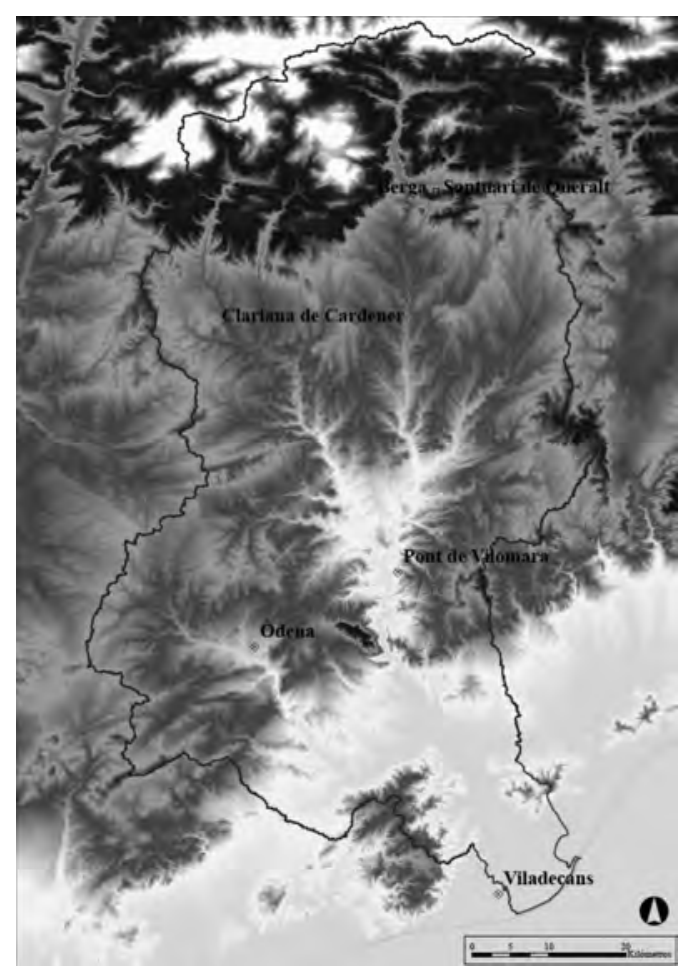

\subsection{Análisis de datos}

Los datos meteorológicos analizados corresponden a las siguientes variables:

- Velocidad $(\mathrm{m} / \mathrm{s})$, dirección $\left(^{\circ}\right)$ y racha máxima del viento $(\mathrm{m} / \mathrm{s})$

- Temperatura $\left({ }^{\circ} \mathrm{C}\right)$ y radiación global $\left(\mathrm{W} / \mathrm{m}^{2}\right)$

- Humedad relativa (\%)

- Precipitación (mm)

- Presión atmosférica (excepto las estaciones de Viladecans y Berga (Queralt))

Los datos disponibles de Viladecans y Berga (Queralt) son horarios, mientras que los de las otras estaciones son semihorarios; ello no afecta a su tratamiento. Respecto a la medida de la velocidad y la dirección del viento, ésta se efectúa a 10 metros de altura excepto en Viladecans, donde se efectúa a 2 metros. Aunque la altura oficial de medición es la primera, se decidió convertir los datos tomados a $10 \mathrm{~m}$ para que correspondiesen a medidas tomadas a $2 \mathrm{~m}$, ya que ésa es una altura ideal para estudios relacionados con incendios forestales. Mediante una consulta al Servei Meteorològic de Catalunya, se aplicó un factor de corrección 
que se usa habitualmente en esos casos, dividiendo el valor tomado a $10 \mathrm{~m}$ por 1,402 .

Para la caracterización de los vientos locales de la cuenca, se analizaron una serie de parámetros siguiendo el hilo del estudio de Cruz (2001) «Estudi de la brisa a Sant Feliu de Llobregat - Juny, juliol i agost de 2000 i 2001» en el que se estudian las principales características de la brisa marina en un punto concreto de la zona baja de la cuenca del Llobregat. Los parámetros para cada estación son la hora de inicio y fin, dirección media, velocidad media, y racha máxima del viento y hora de registro de ésta.

Los criterios aplicados para el filtro de los datos, de forma que se pueda discernir claramente aquello que corresponde al viento local, se basan en los establecidos por Azorín Molina (2004). Son los siguientes:

a) cambio brusco de la dirección y la velocidad del viento al inicio y al final del periodo diurno.

El cambio de dirección es de unos 120 a 180 grados, y se observa la aparición de un viento del segundo o tercer cuadrante, próximo a la componente sur. El umbral mínimo que se establece como velocidad media del viento para que se pueda considerar como viento local de origen térmico es de $5,4 \mathrm{~km} / \mathrm{h}(1,5 \mathrm{~m} / \mathrm{s})$. Normalmente se produce un periodo transitorio de calma y dirección errática. Dado que los datos de viento tomados a 10 metros se han corregido para que sean equivalentes a los datos tomados a 2 metros, lo mismo se ha aplicado al umbral mínimo, de modo que el considerado ha sido de $3,9 \mathrm{~km} / \mathrm{h}(1,1 \mathrm{~m} / \mathrm{s})$.

b) campo bárico indefinido o de escaso gradiente en niveles superficiales sobre el espacio sinóptico de la cuenca del Mediterráneo Occidental y levante de la Península.

Éstas son las situaciones sinópticas ideales, parecidas y compatibles entre ellas:

1) Dominio de las altas presiones, con el anticiclón de las Azores más o menos centrado, bloqueando el paso de frentes y perturbaciones.

2) Situaciones de pantano barométrico, con presiones próximas a la normal y un escaso gradiente bárico afectando todo el Mediterráneo Occidental. Puede existir una cierta situación de bloqueo e indefinición.

3) Situaciones de altas o bajas presiones relativas, como por ejemplo, la baja térmica; situación plenamente estival producida debido al calor y en zonas continentales. A menudo se complementa con una situación de pantano barométrico en la costa.

Para la aplicación de este criterio, se han utilizado los boletines mensuales de junio, julio y agosto del Servei Meteorològic de Catalunya, pudiendo determinar los días con situaciones que no eran claramente estables y por tanto, susceptibles de no 
cumplir la premisa anterior. Una vez establecida la primera selección, se han consultado también los mapas de reanálisis del portal alemán Wetterzentrale. De ello ha surgido una lista de días probablemente inestables, útil como herramienta complementaria en el momento de eliminar aquellos días que no cumplían con un patrón habitual de estabilidad (por ejemplo, debido a precipitación significativa, viento fuerte de carácter sinóptico, viento errático en cuanto a dirección y velocidad...) para cada una de las cinco estaciones. Esta información se ha usado como complemento, ya que la simple observación y análisis de los datos meteorológicos permitía detectar los episodios de situaciones zonales o inestables (Prohom, 1998), y de este modo se ha priorizado la información de carácter local extraída directamente de los datos de las estaciones.

En el estudio de Azorín Molina (2004) aparece un tercer criterio: diferencia térmica positiva entre tierra firme y el mar (aprox. $2^{\circ} \mathrm{C}$ ), que aquí se ha decidido no considerar por cuatro razones. La primera, porque sólo sirve para la brisa marina y no para los vientos locales del interior. La segunda, porque es raro que en un día estable de verano no se cumpla esta condición. La tercera, la poca información disponible de temperatura del agua del mar. Y la cuarta, otros estudios (Prohom, 1998) no lo han considerado; o lo han cuestionado, ya que la brisa marina puede establecerse también aunque no exista esa diferencia térmica positiva (Azorín Molina, 2004).

Por último, juntamente con los crierios (a y b) considerados, la observación de los siguientes elementos (Azorín Molina, 2004) ayuda a la detección de los vientos locales:

1) Leve descenso o amortiguamiento del aumento de temperatura con la entrada de la marinada.

2) Leve aumento de la humedad relativa del aire con la entrada de la marinada.

3) Leve descenso de la presión atmosférica cuando empiezan a soplar los vientos locales diurnos, con un mínimo durante la tarde coincidiendo con las velocidades máximas, y una recuperación hasta los valores iniciales una vez establecida la calma o el periodo transitorio del atardecer de paso a los terrales.

\section{RESULTADOS}

\subsection{Viladecans}

La estación meteorológica de Viladecans muestra un patrón de comportamiento del viento local diurno condicionado por la brisa marina, que sopló de forma regular en un $77,5 \%$ de los días analizados. La velocidad media del viento es de $7,5 \mathrm{~km} / \mathrm{h}$ y la racha máxima de $23,7 \mathrm{~km} / \mathrm{h}$, las más bajas de las estaciones estudiadas. Una vez establecida la brisa marina, se puede observar que la velocidad se mantiene más o menos constante a lo largo de las horas y sólo se llega a una velocidad horaria media máxima de $8,3 \mathrm{~km} / \mathrm{h}$ (véase último gráfico de la tabla y 
figuras 2); hecho que contrasta con los datos de las otras cuatro estaciones, donde en las horas de máxima actividad del viento local se superan, como mínimo, los $10 \mathrm{~km} / \mathrm{h}$. En este mismo gráfico se observa que la dirección habitual es de componente sur, virando ligeramente y a lo largo del día a suroeste. Ello se corresponde con la entrada de la marinada en la costa catalana desviada por el efecto de Coriolis (Redaño et al., 1991). Este viraje también se puede relacionar con el periodo transitorio que se produce al final del día y con el paso a los terrales de componente norte muy flojo. Cabe señalar que algunos días la brisa marina sopla más de levante, con direcciones de sureste y este, probablemente por la influencia de la situación sinóptica general y coincidiendo con los resultados del estudio de la brisa en Sant Feliu de Llobregat de Cruz (2001).

En cuanto al horario, el inicio se produce en un $71,5 \%$ de los días entre las 10-13h, y hasta en uno de cada tres, entre las 10-11h. En algunos casos (6,1\%), la brisa marina se establece después del mediodía solar (14h en horario oficial), fenómeno detectado también en el estudio de la marinada de Sant Pere Pescador de Prohom (1998), y donde se afirma que casi siempre se debe a un retraso en la generación del sistema debido a una situación zonal previa. El fin se produce entre las 20-23h en un 59,3\% de los casos, sin que destaque un periodo de una sola hora. Por último, el horario de la racha máxima es más variado y las tres franjas horarias (14-17h) que cuentan más casos sólo suponen el 44,5\% del total. Ninguna franja horaria llega al 20\% de los días, y en cambio, hasta siete (12-19h) cuentan más del 10\%.

Tabla y figuras 2. Parámetros básicos del viento local diurno en Viladecans ${ }^{1}$.

\begin{tabular}{|c|r|}
\hline Días & 214 \\
\hline \% Días & 77,5 \\
\hline V media $(\mathrm{km} / \mathrm{h})(\mathrm{a} 2 \mathrm{~m})$ & 7,5 \\
\hline Racha máx $(\mathrm{km} / \mathrm{h})(\mathrm{a} 2 \mathrm{~m})$ & 23,7 \\
\hline Dirección media $\left({ }^{\circ}\right)$ & 168 \\
\hline Rumbo & $\mathrm{S}$ \\
\hline Hora inicio & $11: 13$ \\
\hline Hora fin & $21: 43$ \\
\hline Hora racha máx & $15: 26$ \\
\hline
\end{tabular}

1 El gráfico de velocidad y dirección del viento local diurno de cada una de las cinco estaciones muestra los periodos horarios o semihorarios de actividad en que por lo menos se hayan registrado un $25 \%$ de los días seleccionados con patrón de comportamiento habitual del viento local. 

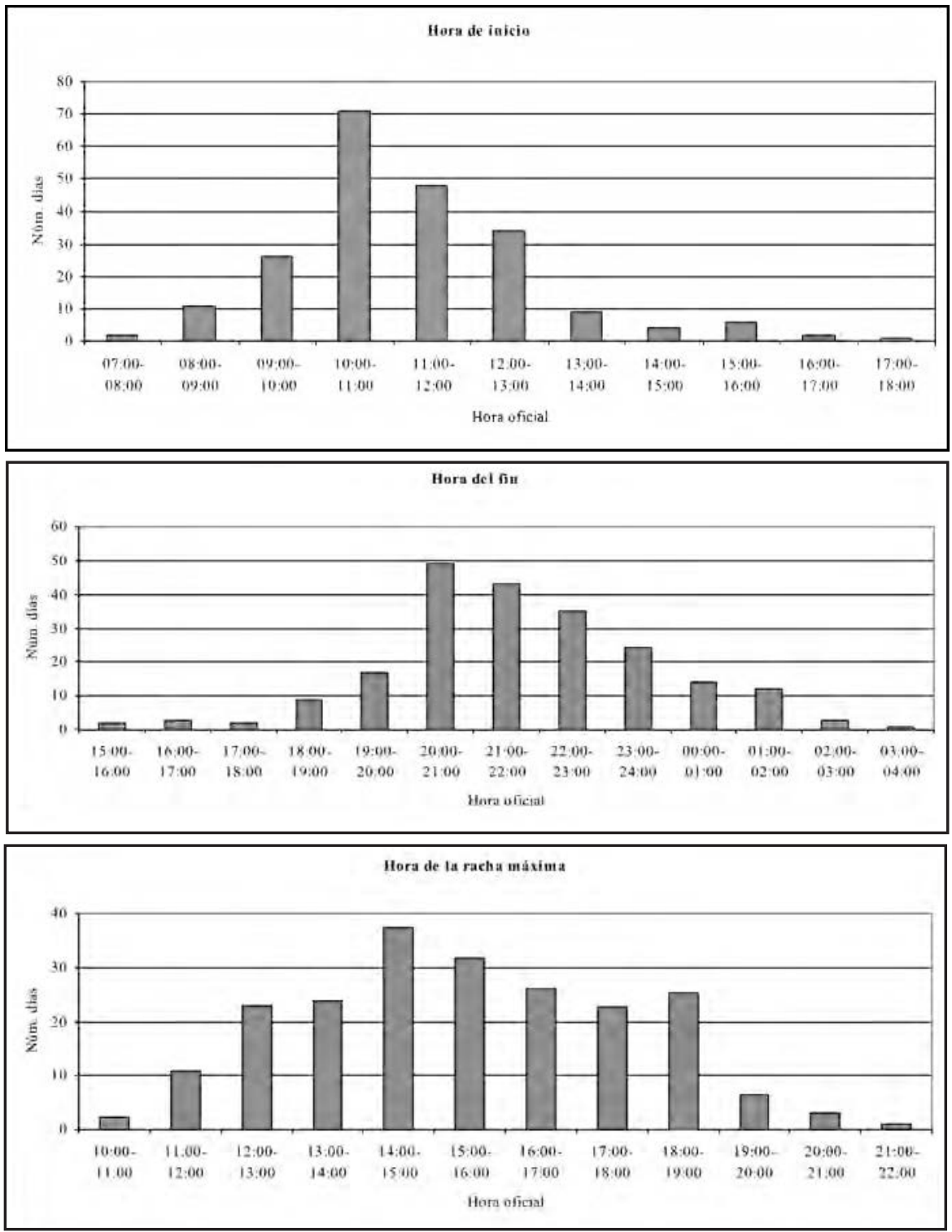


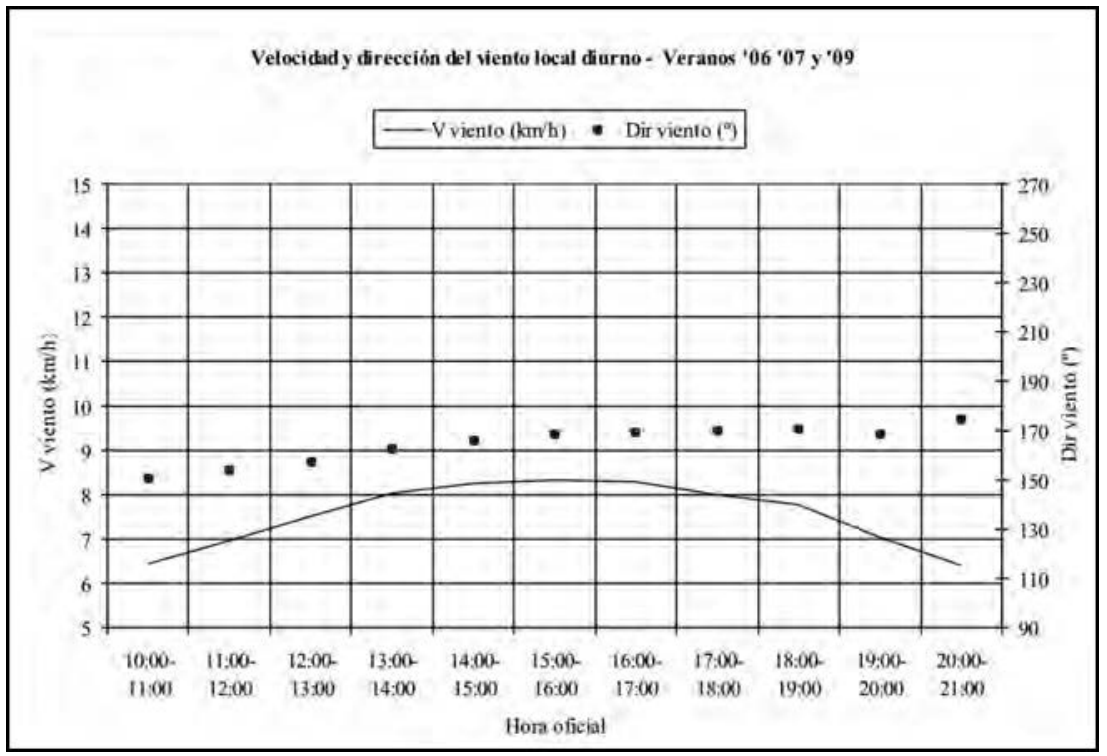

\subsection{El Pont de Vilomara}

La estación del Pont de Vilomara es la segunda de las estudiadas con una velocidad media del viento local diurno más alta $(10,6 \mathrm{~km} / \mathrm{h})$ y aquella donde la racha máxima es más fuerte $(29,2 \mathrm{~km} / \mathrm{h})$, con diferencia la única que se aproxima a los 30 $\mathrm{km} / \mathrm{h}$. En cuanto a las medias horarias, aquí sí que el viento local va ganando fuerza claramente a lo largo del día, y desde los $6 \mathrm{~km} / \mathrm{h}$ del periodo 11-11:30h aumenta continuamente hasta los $14 \mathrm{~km} / \mathrm{h}$ entre las 17:30-18h, para volver a los valores iniciales a las 23-23:30h $(6,1 \mathrm{~km} / \mathrm{h})$. La dirección es de sur, con un ligero viraje continuo durante el día desde el tercer cuadrante (S-W) hacia el segundo (E-S).

En cuanto al horario, todo el sistema (inicio, fin y racha máxima) muestra entre una hora y una hora y media de retraso respecto a Viladecans. Éste es el único elemento que podría llevarnos a pensar que el viento local diurno se explica aquí también por la brisa marina, que iría ganando terreno poco a poco Llobregat arriba. Por el contrario, el análisis horario de las otras estaciones, la geografía de la zona y otros elementos que se detallarán más adelante, descartan esa opción. El inicio se concentra en un $71,2 \%$ de los casos entre las $11-14$ h y destaca claramente la franja horaria de las $11-12 \mathrm{~h}$ con un $34,7 \%$ del total. El fin se produce entre las $22-01$ h en un $68,9 \%$ de los casos con un máximo del $28,8 \%$ de los días entre las 23-24h. La racha máxima también se concentra mucho (71\% de los casos) en un periodo de tres horas, de las 16-19h. El resto, se produce antes de las 16h en un 20,3\% de los días y sólo en un $8,7 \%$ después de las 19 h. 
Por último, se trata de una de las estaciones que muestra un patrón de comportamiento del viento local diurno más homogéneo y que se repite más claramente día tras día en condiciones atmosféricas estables (hasta en un 80,4\% de los días de verano); hecho que probablemente se deba al emplazamiento geográfico, muy próximo al río Llobregat, donde se canaliza el viento de valle, elemento protagonista del patrón de viento local de esa zona del Bages y totalmente compatible con los resultados extraídos en cuanto a velocidad, dirección y horario.

Tabla y figuras 3. Parámetros básicos del viento local diurno en el Pont de Vilomara.

\begin{tabular}{|c|r|}
\hline Días & 222 \\
\hline \% Días & 80,4 \\
\hline V media $(\mathrm{km} / \mathrm{h})(\mathrm{a} 2 \mathrm{~m})$ & 10,6 \\
\hline Racha máx $(\mathrm{km} / \mathrm{h})(\mathrm{a} 2 \mathrm{~m})$ & 29,2 \\
\hline Dirección media $\left({ }^{\circ}\right)$ & 184 \\
\hline Rumbo & $\mathrm{S}$ \\
\hline Hora inicio & $12: 31$ \\
\hline Hora fin & $23: 12$ \\
\hline Hora racha máx & $17: 06$ \\
\hline
\end{tabular}
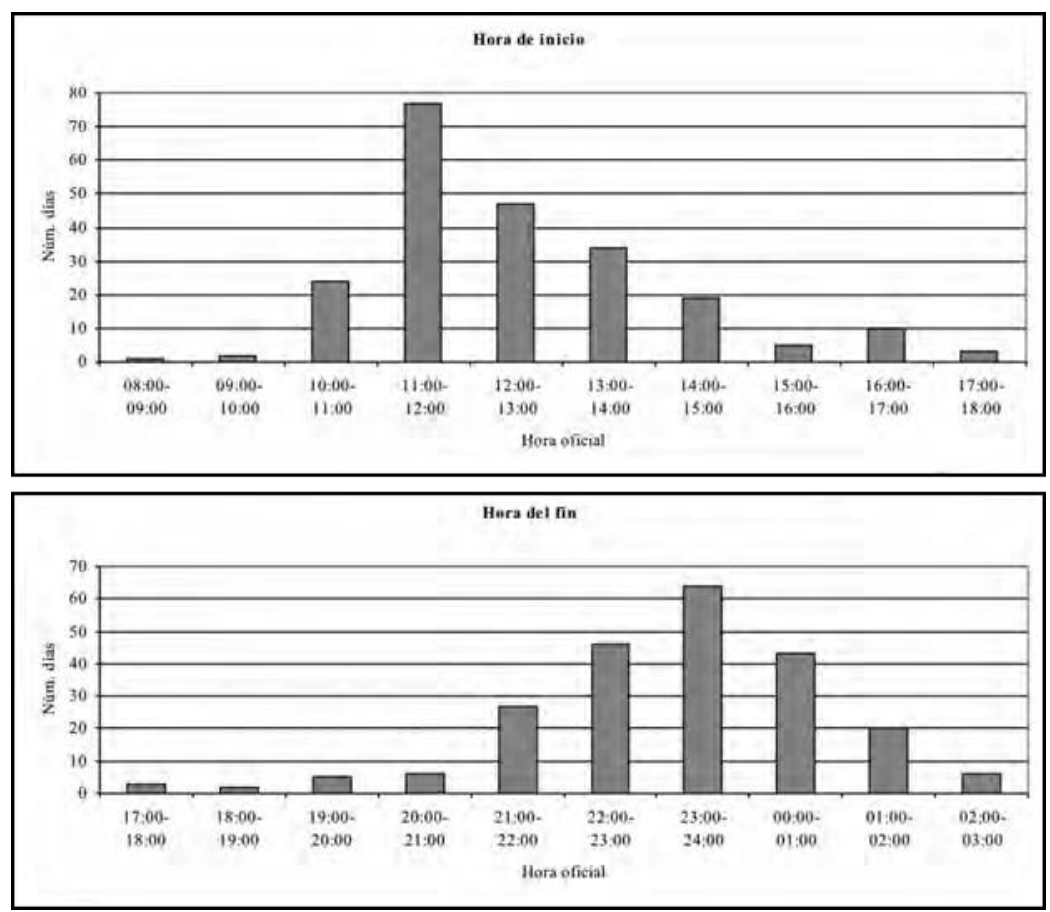

Investigaciones geográficas, nº 58, pp. 267 - 289 

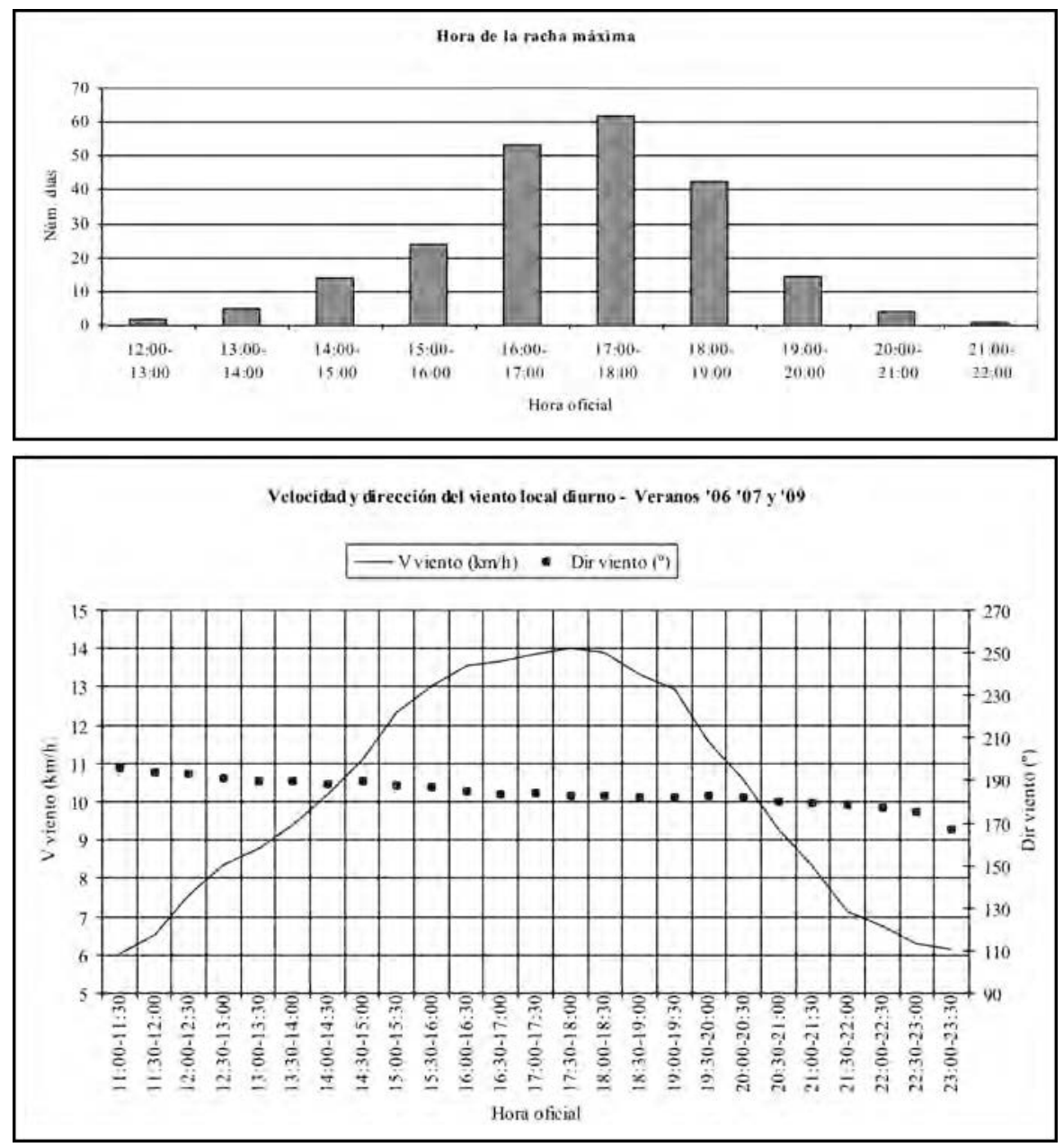

\subsection{Berga (Queralt)}

La velocidad media del viento local diurno en la estación del Santuario de Queralt (municipio de Berga) no es alta comparada con la del resto de estaciones estudiadas $(9,1 \mathrm{~km} / \mathrm{h})$, pero en cambio, la racha máxima llega hasta los 26,6 $\mathrm{km} / \mathrm{h}$, sólo por debajo del valor registrado habitualmente en el Pont de Vilomara. En cuanto a las medias horarias, la velocidad aumenta claramente a lo largo del día y el punto álgido se produce entre las $15-16 \mathrm{~h}$ con $11,8 \mathrm{~km} / \mathrm{h}$, para volver a disminuir hasta los 6 km/h entre las 21-22h. La dirección habitual es de sur, con un ligero viraje hacia suroeste a lo largo del día. 
El inicio del viento local diurno (10:01h de media) es el que se produce más temprano de las cinco estaciones analizadas, más de una hora antes que el inicio medio de la brisa marina en Viladecans. Este hecho, por un lado es uno de los elementos que demuestra que la marinada no se canaliza río Llobregat arriba indefinidamente y no rige el patrón del viento local en Berga (Queralt) y, por extensión, en la parte alta de la cuenca. Por otro, muy probablemente se explica porque el elemento protagonista del patrón de comportamiento del viento local es el viento de ladera. La estación meteorológica se encuentra situada en la sierra de Queralt, en una ladera orientada al sur, y tal y como se ha comentado en la introducción, los vientos de ladera se inician poco después de la salida del sol (antes que el viento de valle) en las laderas orientadas al este y al sur. Además, el ligero viraje de la dirección hacia suroeste a lo largo del día coincide con el movimiento aparente del sol, que pasa a calentar zonas más encaradas hacia el oeste. La hora de inicio se concentra en el periodo $8-11$ h hasta en un $84,0 \%$ de los casos. La hora del fin varía bastante más (el periodo más significativo, entre las 21-24h, concentra sólo un $56,9 \%$ ), sobre todo debido a las tormentas de tarde y dinámicas orográficas propias de una zona elevada $(1140 \mathrm{~m})$ del Prepirineo, que provocan que el fin de la actividad se avance. Y en cuanto a la racha máxima, ésta se concentra entre las 15-18h con un 67,6\% de los casos, destacándose también el periodo $14-15$ h con un $14,9 \%$ del total (valor parecido al del periodo 17 18h: $16,2 \%)$.

Tabla y figuras 4. Parámetros básicos del viento local diurno en Berga (Queralt).

\begin{tabular}{|c|r|}
\hline Días & 225 \\
\hline \% Días & 81,5 \\
\hline V media (km/h) (a 2m) & 9,1 \\
\hline Racha máx (km/h) (a 2m) & 26,6 \\
\hline Dirección media $\left(^{\circ}\right)$ & 180 \\
\hline Rumbo & $\mathrm{S}$ \\
\hline Hora inicio & $10: 01$ \\
\hline Hora fin & $21: 55$ \\
\hline Hora racha máx & $15: 58$ \\
\hline
\end{tabular}



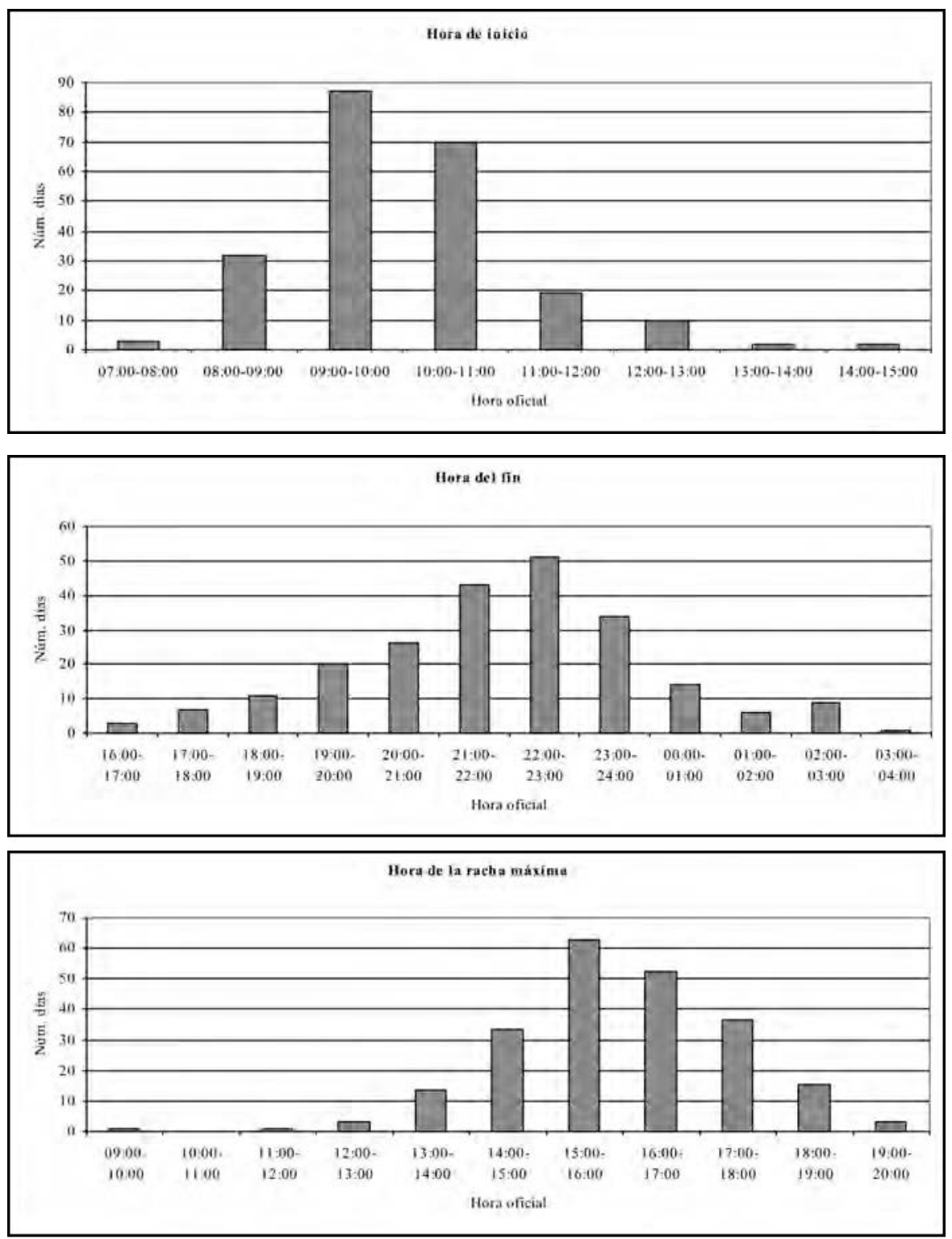


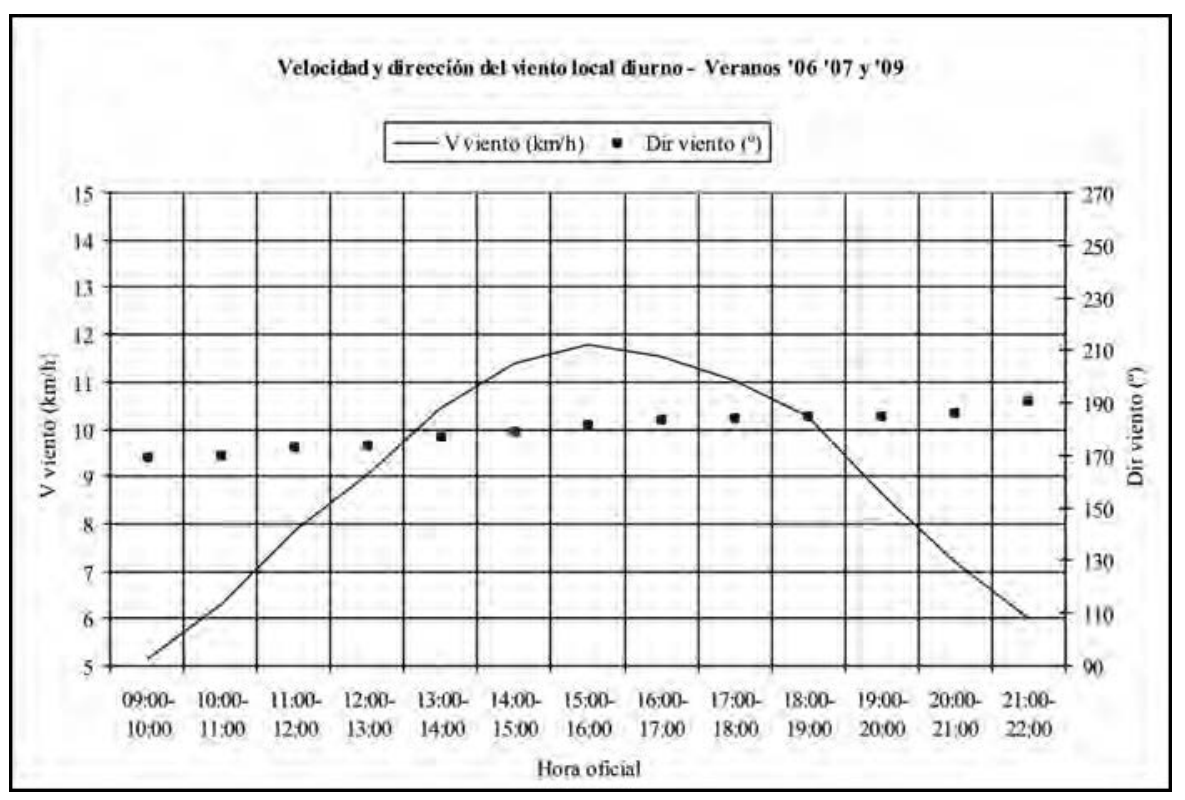

\section{4. Òdena}

La estación meteorológica de Òdena es, de las cinco, la que registra una velocidad media del viento local diurno más elevada: 10,8 km/h. Por el contrario, la racha máxima también es destacable $(26,1 \mathrm{~km} / \mathrm{h})$, pero queda por detrás de las registradas en el Pont de Vilomara y en Berga (Queralt). La dirección habitual es de sureste, en consonancia con la localización geográfica, en el valle del río Anoia, que fluye aproximadamente de noroeste a sureste.

El horario medio de inicio se produce más bien tarde (12:08h) y es parecido al del Pont de Vilomara. Hasta un 72,5\% de los casos se concentran en el periodo de las 10-13h. En cuanto al fin, también se destaca mucho un periodo de tres horas, de 22-01h, con un 77,3\% de los días analizados. La racha máxima se registra en un 70,8\% de los días de verano entre las 15-18h.

Como sucedía en el Pont de Vilomara, el patrón de comportamiento del viento local diurno es muy homogéneo y se repite claramente la mayoría de días de verano, lo que muy probablemente se deba a la localización, próxima al fondo del valle. Por lo tanto, aquí el elemento clave también es el viento de valle; una afirmación avalada no sólo por el emplazamiento geográfico, sino también por los resultados extraídos de velocidad, dirección, horario, y las similitudes con la localización y el patrón observado en la estación del Pont de Vilomara. 
Tabla y figuras 5. Parámetros básicos del viento local diurno en Òdena.

\begin{tabular}{|c|r|}
\hline Días & 211 \\
\hline \% Días & 76,4 \\
\hline V media $(\mathrm{km} / \mathrm{h})(\mathrm{a} 2 \mathrm{~m})$ & 10,8 \\
\hline Racha máx $(\mathrm{km} / \mathrm{h})(\mathrm{a} 2 \mathrm{~m})$ & 26,1 \\
\hline Dirección media $\left.{ }^{\circ}\right)$ & 145 \\
\hline Rumbo & $\mathrm{SE}$ \\
\hline Hora inicio & $12: 08$ \\
\hline Hora fin & $23: 14$ \\
\hline Hora racha máx & $16: 33$ \\
\hline
\end{tabular}
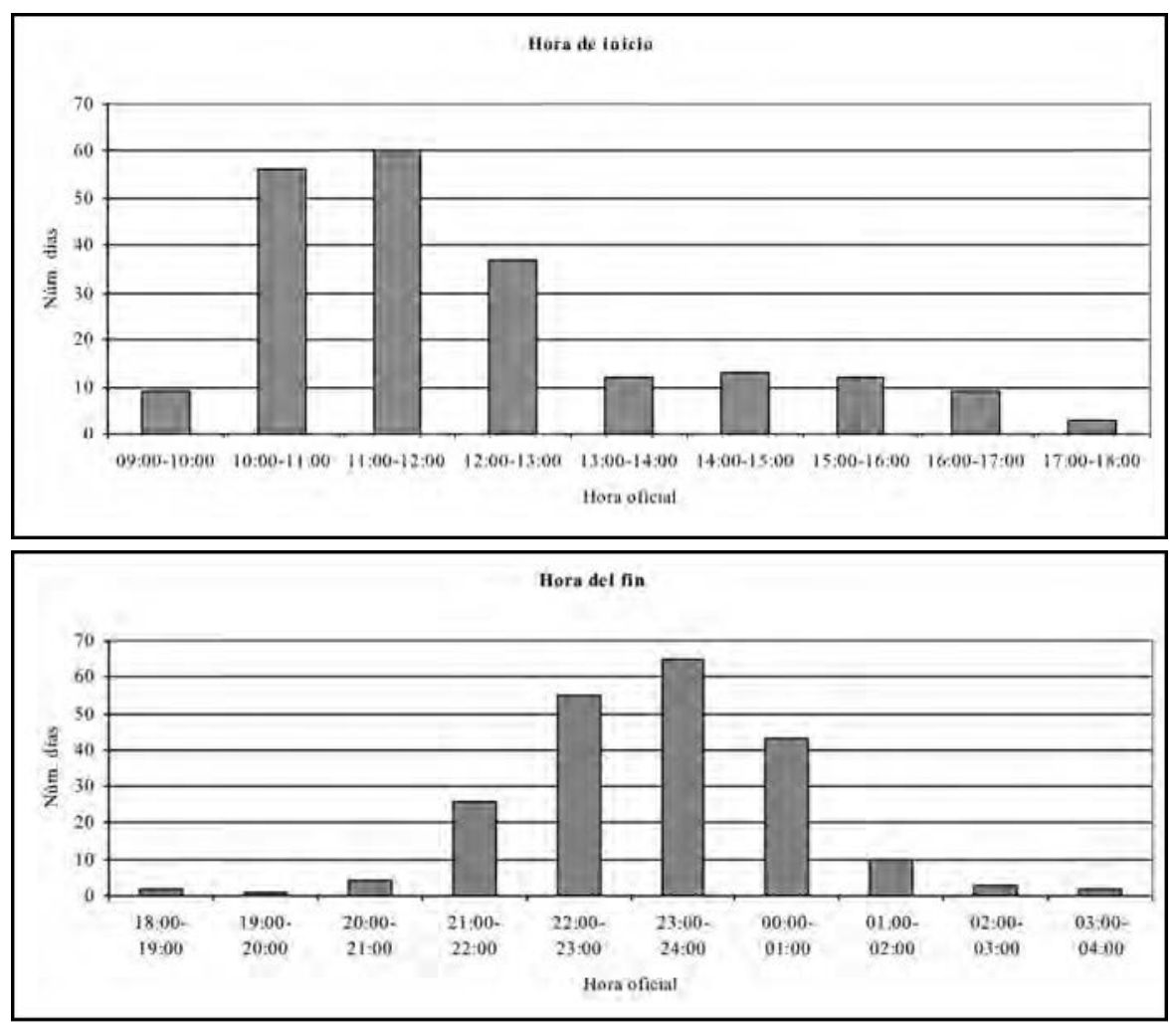

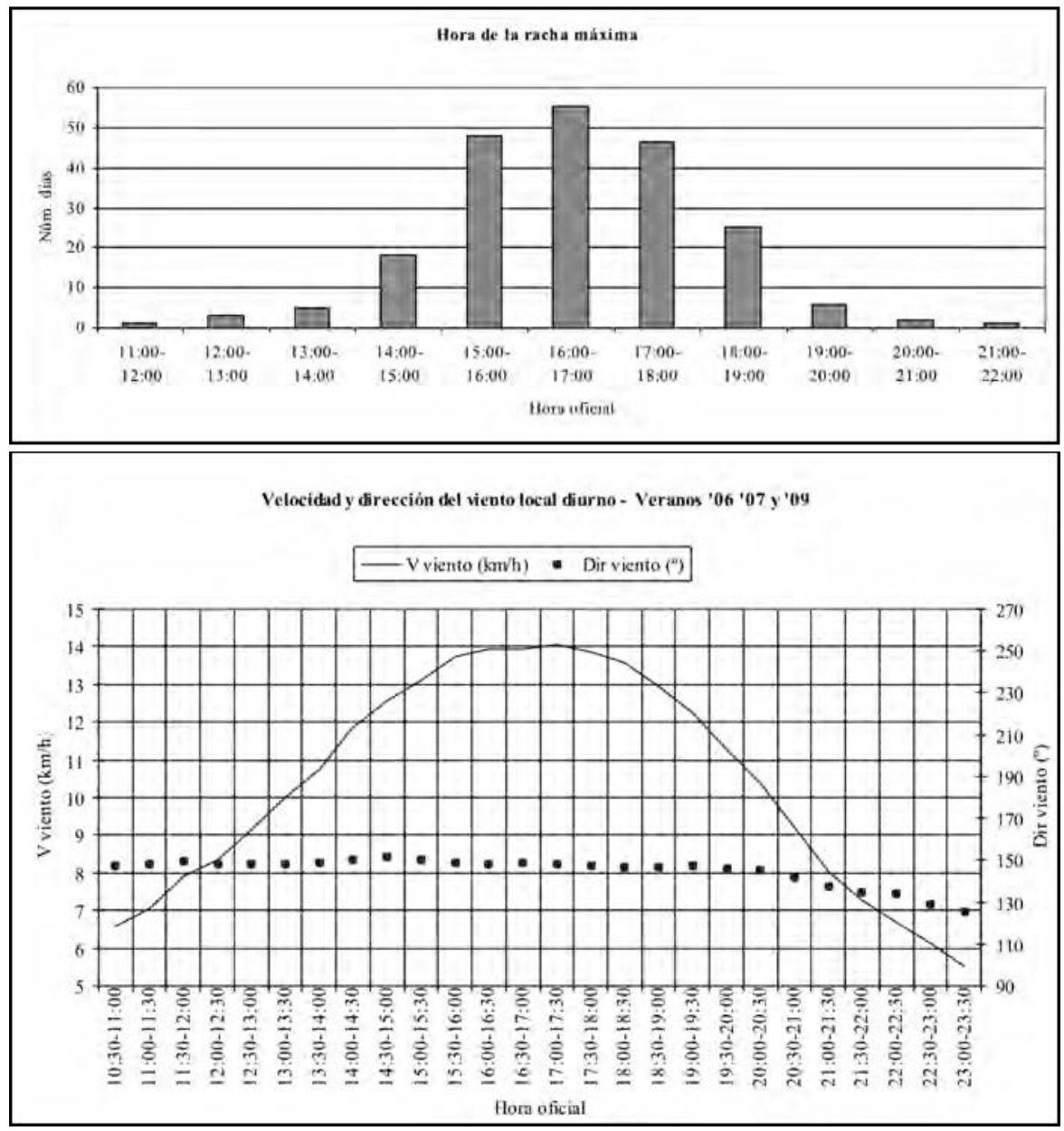

\subsection{Clariana de Cardener}

La estación de Clariana de Cardener registra una velocidad media del viento local diurno de $8,7 \mathrm{~km} / \mathrm{h}$, sólo superior a la que registra la brisa marina en la estación de Viladecans. La racha máxima llega hasta los 25,6 km/h y la dirección habitual es de sur, aunque a última hora de la tarde se observa un claro viraje a sureste (véase último gráfico de Tablas y figuras 6). Eso es debido a que a menudo sopla viento de componente este al final del día; cuya causa (quizá relacionada con la orografía local) no es determinable a partir de los datos meteorológicos disponibles. A pesar de eso, este viento siempre se produce al atardecer, cuando la velocidad disminuye, y no es significativo en comparación con el viento de componente sur que sopla la mayor parte del periodo diurno. 
Del mismo modo que en la estación de Berga (Queralt), la actividad del viento local se detecta, de media, antes (10:50h) que el inicio de la brisa marina en Viladecans (11:13h). En un 73,4\% de los días el inicio se detecta entre las 0912h. En cuanto al fin, este se produce en un 70\% de los casos entre las 21-24h. La racha máxima cuenta un 64\% de los casos entre las 15-18h, destacándose también los periodos $14-15$ h y $18-19$ h con un $11,1 \%$ y un $14,0 \%$ de las rachas máximas diarias detectadas respectivamente.

La velocidad, dirección, horario y las similitudes con los resultados extraídos de los datos de la estación de Berga (Queralt) llevan a pensar que probablemente el factor determinante del patrón del viento local sea el viento de ladera, y no el viento de valle de la cuenca del Cardener. La localización geográfica de la estación, a $693 \mathrm{~m}$ de altitud, unos doscientos metros por encima del núcleo de población al lado del río, permite apoyar esa afirmación.

Tabla y figuras 6. Parámetros básicos del viento local diurno en Clariana de Cardener.

\begin{tabular}{|c|r|}
\hline Días & 207 \\
\hline \% Días & 75,0 \\
\hline V media $(\mathrm{km} / \mathrm{h})(\mathrm{a} 2 \mathrm{~m})$ & 8,7 \\
\hline Racha máx $(\mathrm{km} / \mathrm{h})(\mathrm{a} 2 \mathrm{~m})$ & 25,6 \\
\hline Dirección media $\left.{ }^{\circ}\right)$ & 185 \\
\hline Rumbo & $\mathrm{S}$ \\
\hline Hora inicio & $10: 50$ \\
\hline Hora fin & $22: 25$ \\
\hline Hora racha máx & $16: 26$ \\
\hline
\end{tabular}

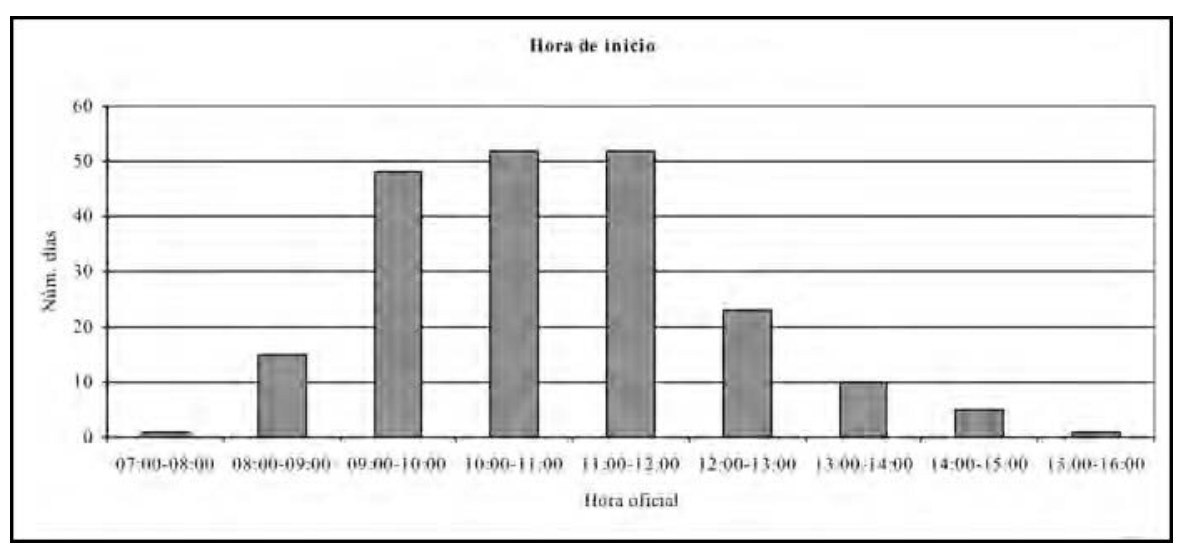



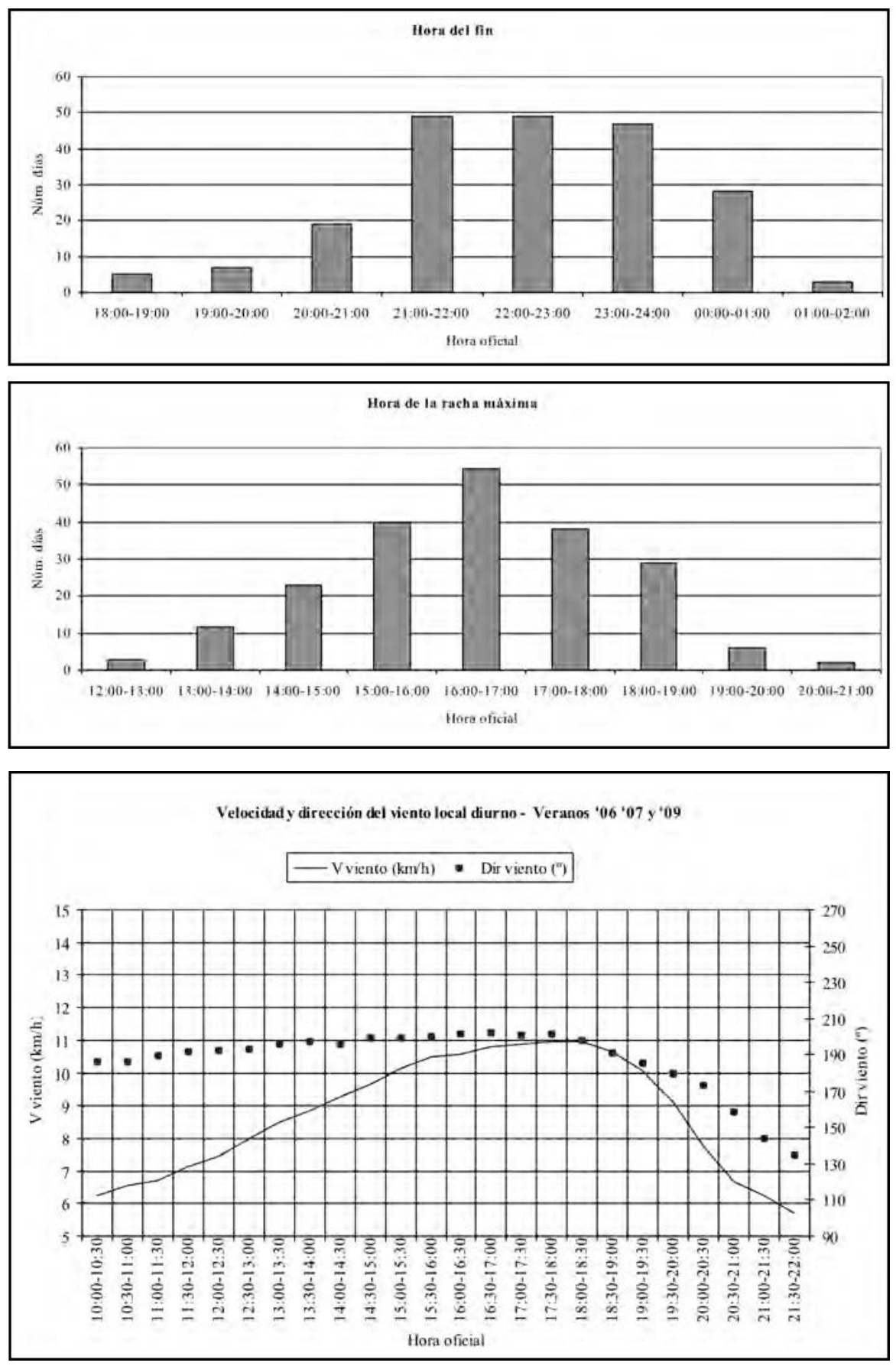

Investigaciones geográficas, nº 58, pp. 267 - 289 


\subsection{Resultados globales}

La hora de inicio habitual de actividad se da por la mañana, unas horas después de la salida del sol, en especial durante el periodo entre las 09-12h, resultado que concuerda con las características de los sistemas del viento local, ya que es necesario cierto tiempo para que se note el efecto de la radiación solar sobre el terreno y se activen el viento de ladera, el viento de valle y la brisa marina. No obstante, la influencia de la situación sinóptica atmosférica provoca que algunos días el inicio se detecte más adelante, durante las primeras horas de la tarde. Lo mismo sucede con la hora del fin, que se concentra en las horas posteriores a la puesta del sol, en especial entre las $21-24 \mathrm{~h}$, aunque a veces la actividad del viento local diurno se prolonga hasta la madrugada (en consonancia con los resultados del estudio de Cruz (2001) sobre la brisa en Sant Feliu de Llobregat) o finaliza por la tarde si se inestabiliza la situación atmosférica (principalmente en la parte alta de la cuenca debido a las tormentas de tarde que se originan en el Pirineo y Prepirineo). En definitiva, en condiciones de estabilidad, el horario de actividad del viento local es, lógicamente, una respuesta ligeramente atrasada del horario de actividad solar.

La velocidad media se sitúa entre las 7 y los $11 \mathrm{~km} / \mathrm{h}$, coincidiendo a grandes rasgos con los resultados de otros estudios realizados en Barcelona (Redaño et al., 1991) y en Alicante (Azorín Molina, 2004), mientras que la dirección media es de componente sur, y en menor medida de sureste o suroeste (sobre todo en función de la posición de los valles donde se ubican las estaciones meteorológicas en el interior).

La racha máxima se sitúa entre los $23-30 \mathrm{~km} / \mathrm{h}$ y cabe destacar que muy pocas veces se superan los $30 \mathrm{~km} / \mathrm{h}$, que se podrían considerar como el umbral límite del viento local en la cuenca del Llobregat (valor corregido a $2 \mathrm{~m}$ de altura, que corresponde a $42 \mathrm{~km} / \mathrm{h}$ si la medida se toma a la altura oficial de 10m). El horario de detección de la racha máxima es variado y ésta prácticamente puede aparecer en cualquier momento del día. No obstante, muy a menudo se produce durante las primeras horas de la tarde, coincidiendo con las velocidades medias más elevadas, y pocas horas después de la temperatura máxima (Azorín Molina, 2002). El horario habitual es entre las 15-18h.

Tabla 7. Características principales del viento local diurno en la cuenca del Llobregat.

\begin{tabular}{|c|c|}
\hline HORA DE INICIO & $09: 00-12: 00$ \\
\hline HORA DEL FIN & $21: 00-24: 00$ \\
\hline VELOCIDAD MEDIA & $7-11 \mathrm{~km} / \mathrm{h}$ \\
\hline DIRECCIÓN MEDIA & $\mathrm{S}(\mathrm{SE}, \mathrm{SO})$ \\
\hline RACHA MÁXIMA & $23-30 \mathrm{~km} / \mathrm{h}$ \\
\hline HORA DE LA RACHA MÁXIMA & $15: 00-18: 00$ \\
\hline
\end{tabular}


Los resultados obtenidos y la bibliografía, que explica como la brisa marina tiene serias dificultades para llegar a zonas interiores si tiene que cruzar obstáculos orográficos (Azorín Molina, 2002), indican que el rol principal de ésta en el comportamiento del viento local se acabaría, probablemente, en la sierra Prelitoral. En el interior, en cambio, el rol principal hay que buscarlo en el viento de valle o en los vientos de ladera, teniendo en cuenta que cada subcuenca forma su propio régimen de vientos. Esta afirmación viene reflejada en el mapa de zonas homogéneas de régimen de incendio forestal del estudio de Castellnou et al. (2009) de tipificación de incendios forestales en Catalunya. En él se puede comprobar como una parte destacada de los incendios topográficos registrados en la zona baja de la cuenca del Llobregat son del subtipo litoral (el elemento de propagación es la brisa marina). Una vez superada la sierra Prelitoral, los incendios topográficos registrados en el resto de la cuenca son sólo del subtipo estándar (el elemento de propagación es el viento de ladera) o próximo a valle principal (el elemento de propagación es el viento de valle).

Si la marinada alcanzase las zonas interiores de la cuenca, de un lado, se registrarían cambios significativos en dos variables meteorológicas con su entrada (una pequeña disminución de la temperatura o amortiguamiento de su aumento, y un pequeño aumento de la humedad relativa), lo cual no se observa con datos horarios o semihorarios a lo largo del día (sólo se observa, precisamente, en la estación costera de Viladecans). Por otro lado, se establecería una gradación en el horario de inicio, empezando a soplar primero en la costa y alcanzando poco a poco el interior, lo cual ha quedado descartado: Viladecans (11:13h), Berga (Queralt) (10:01h) y Clariana de Cardener (10:50h).

Además, se ha comprobado que en jornadas de inestabilidad y lluvia en la costa, y estabilidad y sol en la parte alta de la cuenca (p.ej.: 9 de julio de 2009), en las que claramente no se ha formado la brisa marina; en el norte, el patrón de viento continuaba siendo el mismo de los días estables en toda la cuenca. Por lo tanto, sin poder descartar que la marinada alcance alguna zona interiores de la cuenca del Llobregat, se puede afirmar rotundamente que no es un elemento importante que explique el patrón del viento local más allá de la sierra Prelitoral.

En definitiva, el sistema de vientos locales (marinada-terral, valle-montaña y ladera catabáticos-anabáticos) que se da en la cuenca del Llobregat no se puede considerar un elemento de importante riesgo de propagación de incendios forestales, ya que a menudo es previsible, no alcanza velocidades elevadas y no acostumbra a registrar cambios inesperados de velocidad y dirección. No obstante, sí que supone un factor añadido al riesgo de propagación, ya que las 
velocidades más destacadas ${ }^{2}$ se alcanzan en horas de mucho riesgo: a la temperatura elevada y la humedad baja hay que sumarle, por lo tanto, una velocidad del viento máxima.

\section{CONCLUSIONES}

Los resultados obtenidos demuestran que la brisa marina, entendida como un flujo que desde la costa se canaliza río Llobregat arriba, no explica el patrón del viento local diurno de la cuenca bajo condiciones de estabilidad estival más allá de la sierra Prelitoral. El patrón responde no sólo a la brisa marina en la zona costera, sino también al viento de valle y a los vientos de ladera, en especial, en el interior, teniendo en cuenta que cada subcuenca forma su propio régimen de vientos.

El patrón se caracteriza por un ciclo diario común que se repite día tras día en verano y con una situación atmosférica estable: viento que aumenta su velocidad progresivamente durante el periodo diurno y con una dirección básicamente de componente sur, y que alcanza su máximo durante la tarde, pocas horas después de los máximos de temperatura y radiación solar, momento en el que a menudo se registra también la racha máxima diaria. Al atardecer, el viento disminuye su velocidad, hasta encalma, y vira a direcciones de componente norte.

Mediante la localización geográfica y los resultados de velocidad, dirección y horario del viento local diurno se ha determinado que el elemento principal que rige el patrón de éste en cada una de las estaciones es la brisa marina en Viladecans, el viento de valle en el Pont de Vilomara y Òdena, y el viento de ladera en Berga (Queralt) y Clariana de Cardener. Las estaciones influenciadas por el viento de valle muestran un ciclo diario más homogéneo y más fácil de detectar, hecho explicable muy probablemente por el propio emplazamiento geográfico cerca del fondo de valle y a tocar del río.

En último lugar, se puede afirmar que el sistema de vientos locales que se da en la cuenca del Llobregat no es un elemento de importante riesgo de propagación de incendios forestales por sí mismo, pero sí que supone un factor añadido, en especial porque las velocidades más elevadas se alcanzan en horas de mayor riesgo meteorológico: a la alta temperatura y la baja humedad hay que sumar, por lo tanto, una velocidad del viento máxima.

2 Las velocidades medias en intervalo horario o semihorario, durante las horas de mayor riesgo, casi alcanzan los $15 \mathrm{~km} / \mathrm{h}$, umbral a partir del cual el factor viento supone un riesgo alto (3) en el índice de riesgo meteorológico de propagación de incendios forestales de la Diputació de Barcelona. 


\section{AGRADECIMIENTOS}

La realización de este estudio ha sido posible gracias a la colaboración y apoyo del personal de la Oficina Tècnica de Prevenció Municipal d'Incendis Forestals (OTPMIF) de la Diputació de Barcelona: Ignasi Castelló, Miquel Àngel Villamuera, Jose Luis Herrera, Pilar Raïch, Artur Moncau y Ramon Riera; y complementado con las aportaciones de José Antonio Vázquez (becario de la Oficina), César Azorín Molina (investigador de la Universidad de Alicante) y Aaron Schliem.

\section{BIBLIOGRAFÍA}

Azorín Molina, C. (2002): «La formación de frentes de brisa activos en la comarca alicantina del Alto Vinalopó. El episodio atmosférico de 27 de abril de 2001», en Investigaciones Geográficas, 29, pp. 109-130.

Azorín Molina, C. (2004): «Estimación de la ocurrencia de la brisa marina en Alicante», en García Codron, J. C.; Diego Liaño, C.; Fdez. De Arróyabe Hernáez, P.; Garmendia Pedraja, C.; Rasilla Álvarez, D.; El clima entre el Mar y la Montaña. Asociación Española de Climatología y Universidad de Cantabria, Serie A, nº , Santander.

Azorín Molina, C.; Martín-Vide, J. (2007): «Methodological approach to the study of the daily persistence of the sea breeze in Alicante (Spain)», en Atmósfera 20(1), pp. 57-81.

Castellnou, M.; Pagés, J.; Miralles, M.; Piqué, M. (2009): «Tipificación de los incendios forestales de Cataluña. Elaboración del mapa de incendios de diseño como herramienta para la gestión forestal». $5^{\circ}$ Congreso Forestal Español. Montes y sociedad: saber qué hacer. Ávila.

Cruz, J. (2001): «Estudi de la brisa a Sant Feliu de Llobregat. Juny, juliol i agost de 2000 i $2001 »$. Inédito.

Montserrat, D. (1998): «Situaciones sinópticas relacionadas con el inicio de grandes incendios forestales en Cataluña», en Nimbus, núms. 1 y 2, pp. 93-112.

Olcina Cantos, J.; Azorín Molina, C. (2004): «The meteorological importance of seabreezes in the Levant region of Spain», en Weather, Octubre 2004, vol. 59, n. ${ }^{\circ}$. 10.

Prohom, M. J. (1998): «Principales características de la brisa marina estival en un punto de la costa catalana: Sant Pere Pescador». VIII Coloquio del Grupo de Métodos Cuantitativos de Información Geográfica y Teledetección, AGE, Bellaterra, Barcelona, pp. 167-179.

Redaño, A.; Cruz, J.; Lorente, J. (1991): «Main features of the sea-breeze in Barcelona», en Meteorology and Atmospheric Physics, vol. 46, pp. 175-179.

Salvador, R.; Millán, M. (2003): «Análisis histórico de las brisas en Castellón», en Thethys. Asociación Catalana de Meteorología, pp. 37-51. 